\title{
Validation of a Distributed Energy Management Approach for Smart Grid Based on a Generic Colored Petri Nets Model
}

\author{
Zeineb Mhadhbi, Sajeh Zairi, Cedric Gueguen, and Belhassen Zouari
}

\begin{abstract}
This paper proposes a distributed energy management approach that aims to manage residential energy. The proposed approach considers the neighborhood energy surpluses in order to decrease energy costs. This management is improved based on a set of trust factors that a buyer defines on his neighbors to avoid non-optimal energy purchases. To evaluate the performance of this approach and to study its impact on the neighborhood, we elaborated a formal model representing the overall behavior of the system using colored Petri nets (CP-nets). The choice of CP-nets is justified by the fact that, in our approach, we have to deal with a variety of protocols and several operations that are executing simultaneously, and potentially interacting with each other. This formalism allowed us to formally verify the proposed approach as well as to generate a set of performance indicators. The obtained indicators prove the effectiveness of our solution compared to other approaches.
\end{abstract}

Index Terms-Modeling, smart grid, colored petri nets, formal validation, energy management.

\section{INTRODUCTION}

Motivating consumers to actively and voluntarily participate in such management programs is one of the key challenges of successful energy management schemes in Smart Grids (SGs) [1]-[3]. In this context, various energy management schemes have been proposed [1]-[13]. The main goal of these approaches is to minimize energy costs either by shifting their load at peak hours or by sharing energy in neighborhood.

Some of these existing approaches have considered the management of the surplus energy at home (locally) and at neighborhood [9], [11]-[13]. Indeed, sharing energy between neighbors allows for better profitability. The sharing of energy between neighbors is based on estimation of energy production and consumption. The seller, based on advance estimation to determine his surplus of energy, may confirm to sell a quantity of energy which will not be available later. Based on these uncertain inputs, the buyer accepts to buy a

Manuscript received September 17, 2016; revised April 29, 2017.

Z. Mhadhbi and B. Zouari are with the Mediatron Research Laboratory, Higher School of Communications, University of Carthage, 2083, Ariana, Tunisia (e-mail: zaineb.mhadhbi@gmail.com, belhassen.zouari@supcom.tn).

S. Zairi is with the Higher School of Digital Economy, University of Manouba, 2010 Manouba, Tunisia, and also with the Mediatron Research Laboratory, Higher School of Communications, University of Carthage, 2083, Ariana, Tunisia (e-mail: zairisajeh@fst.rnu.tn).

C. Gueguen is with the Institut de Recherche en Informatique et Syste'mes Ale'atoires Laboratory, University of Rennes1, 35000 Rennes, France (e-mail: cedric.gueguen@irisa.fr). quantity of energy from his neighbors which could not be available at the requested time slot. To overcome this sort of inconvenience, this paper proposes a new solution based on a trust factors that a buyer defines on each neighbor in order to avoid bad (non-optimal) purchases.

In addition, the optimization of grid energy becomes a complex problem requires modeling the overall behavior of SG system [14]. In order to observe the global behavior of the studied SG, a global model representing the different SG components and the different modes of communication between these components is needed [14]. The modeling formalism used should be intuitive and should support specifying appropriate abstraction level. In this paper, we advocate the use of Colored Petri Nets (CP-nets) [15] for the formal specification, simulation, and further analysis of SGs behavior. The existing CP-nets formal methods, specifically the state space generation and handling, may be exploited in order to formally verify the specified system as well as to generate a set of performance indicators. Depending on the obtained indicators, we may support our solution compared to other existing approaches.

The paper considers the simulation of SG system using CP-nets. Specifically, the aim of the paper is to conduct simulation-based performance analysis of a SG approach where the local buying of energy takes into account the trust factor of the neighbor capturing how reliable each neighbor have been in delivering the promised amount of energy. The paper introduces the SG domain and presents the construct $\mathrm{CP}$-nets model which captures the various components in the smart grid system, including the buying algorithm taking into account trust factors. The constructed CP-nets model is then use to compare the trust-factor-based approach to alternative approaches demonstrating the benefit the suggested approach. This paper is organized as follows. Section II introduces the proposed approach. Section III presents the proposed generic CP-nets model representing the SG behavior. Section IV reports simulation results. Section V concludes the material.

\section{Distributed ENERGy MANAGEMENT APPROACH (DEM)}

Proposed contribution called DEM enables to minimize the energy bill of each home by satisfying any demand. Based on DEM, the user begins by querying his local energy, in case local energy shortage, he requests his neighbors and at worst he demands to Grid.

However, such estimation may be incorrect. Thus, a neighbor (a Smart Meter (SM)) may confirm to sell a quantity of energy, for the requested time slot, which will not be wholly available later. The difference between the 
estimation and the real quantity is due to two constraints. The estimated consumption is lower than real consumption. In this case, the seller will decide to let the promised quantity of energy for himself. Or, the estimated production is higher than the produced (due to bad weather). In these cases, the user (buyer) may confirm to buy a quantity of energy which will be not wholly available later. To overcome this sort of inconvenience, each user (SM) must define a trust factor $(t f)$ on each neighbor to specify approximately the energy that can be received later. The considered trust factor on each neighbor allows a better profitability side home. Indeed, based on the considered trust factor, each user may decrease his energy cost by trading with sellers on whom he trusted since they honor their promise in order to avoid non-optimal purchases.

If $t f=0 \%$, the buyer is certain that proposed quantity of energy will not be available. Otherwise, if $t f=100 \%$, then it is certain that proposed quantity of energy will be available and will be received. Trust factor on each neighbor is calculated according to the historical of the previous Right Received amount (RR) divided by the Total quantity Confirmed (TotC) at the requested Time Slot (TS).

$$
t f=\frac{\sum R R}{\sum \operatorname{Tot} C} * 100
$$

Furthermore, the proposed DEM enables to maximize the gain of each home. Every user (by his SM) may periodically decide to propose to sell a surplus of energy (which is not required). He sends to all neighbors a sale request. If there are not any responses, he decides to sell the surplus of energy to Grid.

The proposed DEM approach encourages the cooperation between these neighbors to ensure their profits (gains maximization and cost minimization) by interacting their sub-components (Smart Meter (SM), Energy Management Unit (EMU), Local Energy source/generator (LE)) with each other [14]. In [14], we summarize the different functions and interaction of the different component composing a home (user).

Based on the enumeration of the sub-component' functions, it is easy to show that each component has a generic behavior. It is also easy to show, that there is a lot of interaction and synchronization between the components. Thus, the behavior of a home is defined through a synchronization of all its sub- components behaviors. In the same way, the behavior of a grid is defined through a synchronization of all its homes' behaviors. Hence, modeling separately each sub-component behavior and then interfacing them seems to be a practical method to obtain the global model of the SG. Subsequently, the global model is built more easily.

CP-nets was chosen as a formalism to model the behavior of the Grid as it allows the development of a generic model that may be instantiated based on colored tokens. Also CP-nets enables the modelling of several concurrent operations using shared energy.

\section{CP-NETS MODELS OF DEM SUB-COMPONENTS}

Fig. 1 represents the generic model of a home. This model is based on two typical iterations that will be repeated as many times as the detailed behavior of a given demand requires.

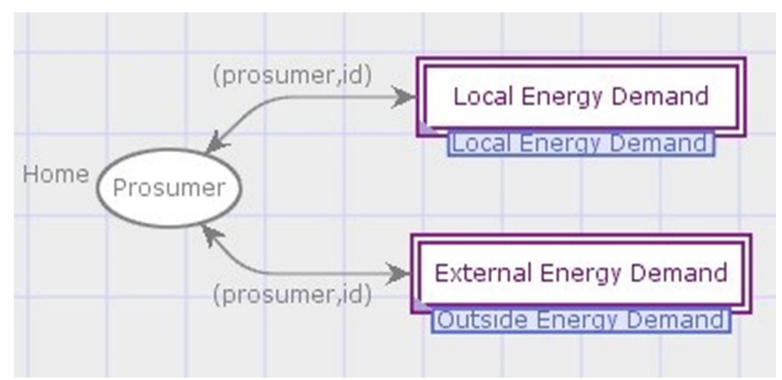

Fig. 1. The generic model of a home.

Such iterations aim to satisfy a received request either for a local or for an outside energy demand. In Fig. 1, the macro transition «Local Energy Demand» models the decision making process when it receives a local demand in order to minimize the cost. The macro transition «External Energy Demand» models the decision making process when it receives an outside demand in order to maximize the gain.

For each substitution transition, there are CP-net sub-models that describe its achievement.

Fig. 2 represents a generic model of the sub-component $\mathrm{SM}$ when it waits for responses (offers) from its neighbors during a definite time interval. This model is based on a generic iteration which is triggered when the place «SM Receives response» includes a token which represents an energy response already sent by its neighbors. This token specifies the available energy and the selling price proposed by a neighbor for the time slot requested. When the timer expires and if the SM hasn't received any response (the place «SM Receives response» is empty), it sends a «START» to the EMU and the EMU sends a «Start» to the appliance. SM sends an energy request to the Grid when the EMU needs the energy immediately; else it waits deals from neighbors during a definite time interval. However, if there is at least one response, according to the neighbors' responses and confirmations, the SM will choose the best alternative to satisfy its needs based on their trust factor. The best alternative is determined by solving an optimization problem considering different criteria such as selling price, available energy. For this, SM sorts in a list all neighbors' responses in decreasing order according to their trust factor. If the quantity of the first seller (presented in its list) is insufficient to accommodate the demand, then SM trades with following seller and so forth. SM satisfies its need by favoring to trade with sellers on whom he trusted much since they honor their promise. In this case, the firing of the transition «SM Sends a MSG Valid to seller» allows sending an response to each chosen neighbor producer to confirm the amount of energy that it should provide for him. It will also send a «START» to the EMU. The choice of the best alternative is made based on the considered energy management approach.

\section{A. Example}

In order to instantiate the CP-net models, we consider a SG which is composed of five prosumers (User 1, User 2, User 3, User 4, and User 5) (see Fig. 3).

We consider three types of profiles that can exist in a week (7 days). We assume that User 1 and User 2 consume, on 
average, more than they produce, User 3 consumes, on average, the same quantity of energy as he produces, User 4 and User 5 consume, on average, less than they produce. We assume that the grid's Buying Price BP/unit $=\$ 0.21$ and the grid's Selling Price SP/unit $=\$ 0.5$, with unit $=100$ Watts $(\mathrm{W})$ $=0.1$ kilowatts $(\mathrm{kW})$. Each user sells his surplus energy at a selling price which is greater than or equal to his cost generation. The cost generation of each user depends on his profile (the one who produces much greater cost): Cost user1 $_{\text {ins }}$ $=\mathrm{BP}+s=2.3$, Cost $_{\text {user } 2}=2.95$, Cost $_{\text {user } 3}=3.6$, Cost $_{\text {user } 4}=$ 4.25 , Cost user $5=\mathrm{SP}-\mathrm{s}=4.9$. This information is presented on our model by specific tokens of the place «Energy selling price» in Fig. 4.

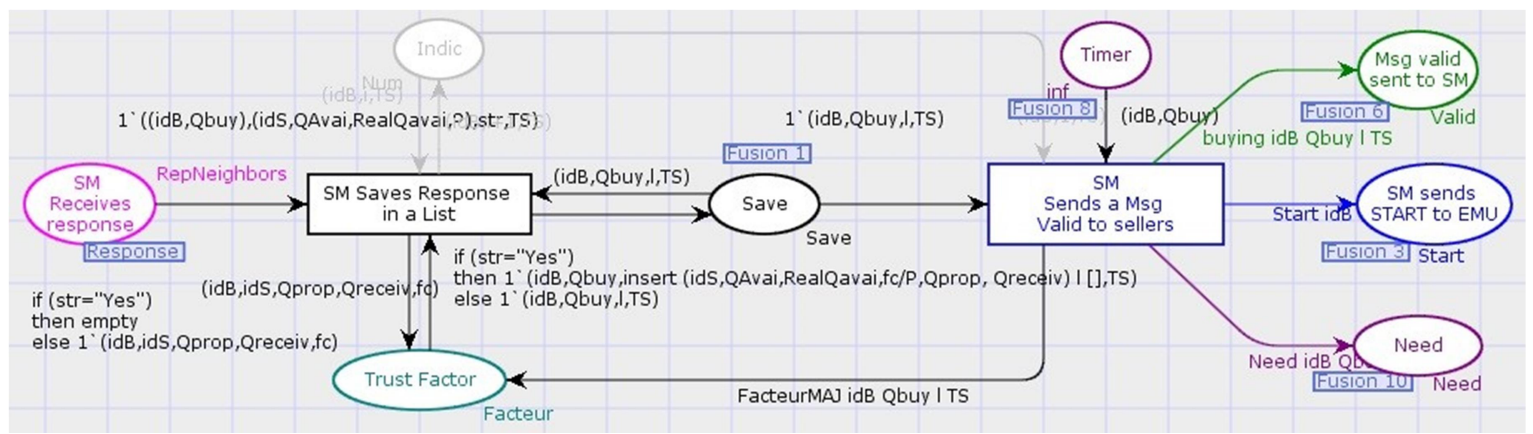

Fig. 2. The generic model of the sub-component (SM) when it receives request energy from SM.

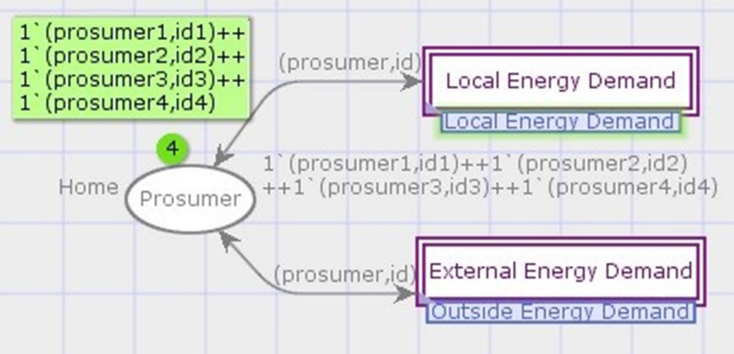

Fig. 3. Tokens in the place Posumer.

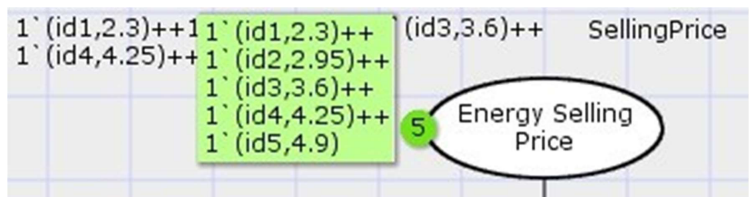

Fig. 4. Tokens in the place Energy selling price.

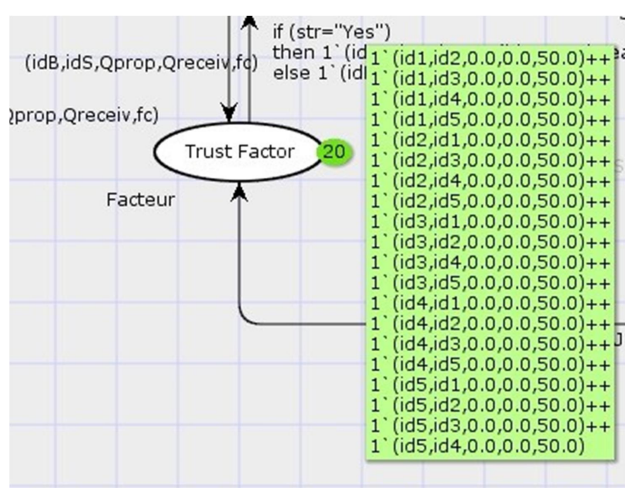

Fig. 5. Tokens in the place «Trust factor».

At first, as no sale is already done, every user initializes at $50 \%$ the trust factor on each neighbor ( $t$ fuseri->Userj $=$ $50 \%$, if $=j$ where $t f$ Useri-> Userj represents the trust factor of the User Un $_{i} U_{s e r}$ ) (Fig. 5). Each tfuseri->Userj will decrease or increase over the time based on the honor of the User $j$ on his promise.

We assume that the consumption $C_{n} \in[1.5 \mathrm{~kW}, 4.5 \mathrm{~kW}]$ for each user. As production $(P)$ depends on the consumption then: $P_{1} \in[0.5 \mathrm{~kW}, 1.5 \mathrm{~kW}], P_{2} \in[1 \mathrm{~kW}, 3 \mathrm{~kW}], P_{3}[1.5 \mathrm{~kW}$,

\section{$4.5 \mathrm{~kW}], P_{4} \in[2 \mathrm{~kW}, 6 \mathrm{~kW}], P_{5} \in[2.5 \mathrm{KW}, 7.5 \mathrm{KW}]$.}

The developed CP-net model was instantiated through the initial marking of a subset of places to be adapted to the studied SG specificities. After simulating the model, we may generate its associated state space. Generating state space associated with the $\mathrm{CP}$-net represents all possible decisions and communication scenarios during 7 days in which we may verify the state of our system such as a deadlock (the neighbors cannot share energy between them). Many analysis techniques are based on state space that can be used to verify behavioral properties.

\section{B. Formal Verification and Validation}

The proposed CP-net model allows formal verification of two main properties of the studied SG. This verification may be performed based on the occurrence graph (O.G, state space) of the studied CP-net, which is directly generated by CPN Tools [16]. Hence, generating the O.G associated with the CP- net allows for obtain all possible scenarios. Also, it will be exploited in order to formally verify the:

1) Safety property

2) Mutual exclusion:

Prevents simultaneous access to a shared resource «LEn» (where $\mathrm{n}$ is the index of user), i.e, only one applicant may access a shared resource $\left\langle L E_{n}\right\rangle$ at a specific moment. Such verification is important, as conflict access may induce to incorrect energy demand decision.

3) Satisfied energy demand:

Any energy demand will be satisfied. Such verifica- tion may be done by comparing the number of tokens in the place «Consumer Request» (which represents the energy demands to satisfy in the initial marking) and the number of tokens in the place «Start» (which represents the already satisfied demands in the final reached marking).

4) Liveness property Based on the state space, we must check after the expired time that the place «Receive Buying Request» does no more include «Demand».

Such validation proofs the correct process of the component through the absence of deadlock and through the satisfaction of all considered demands in a finite time. 
Indeed, the state space may also be exploited in order to generate a set of performance indicators (expense, gain, quantity sold, energy source) which present the simulation results of our example. For our example, we can obtain from the final state marking these indicators. Such information may allow designer to choose the best energy management approach based on the studied SG specificities. In order to prove the effectiveness of our solution, we compare to other existing approaches.

In the following section, we present simulation results and assess the performance of our proposed approach based on the example presented in Section III.A

\section{PERFORMANCE ANALYSIS AND SIMULATION}

The main difference of our proposed approach (DEM) compared to the advanced solution of the literature is the consideration of a trust factor on each neighbor. In order to prove the effectiveness of our solution, we illustrate in this section a comparison, based on the example presented in Section III.A., of our approach with MAS [13] and iHEM [4].

In our simulation model (see Section III.A), we assume that each home has a randomly selected combination of his loads. The latter is used at different times of the day by taking into account that the energy demand is higher in the evening and lower during the night.

Contrary to assumption considered by [13], we assume a realistic hypothesis where the demand and supply are not remaining constant over the time but they vary with the weather, as changes in temperature and humidity affect the demand. With this realistic assumption our proposed trust factor is necessary to avoid all bad purchases.
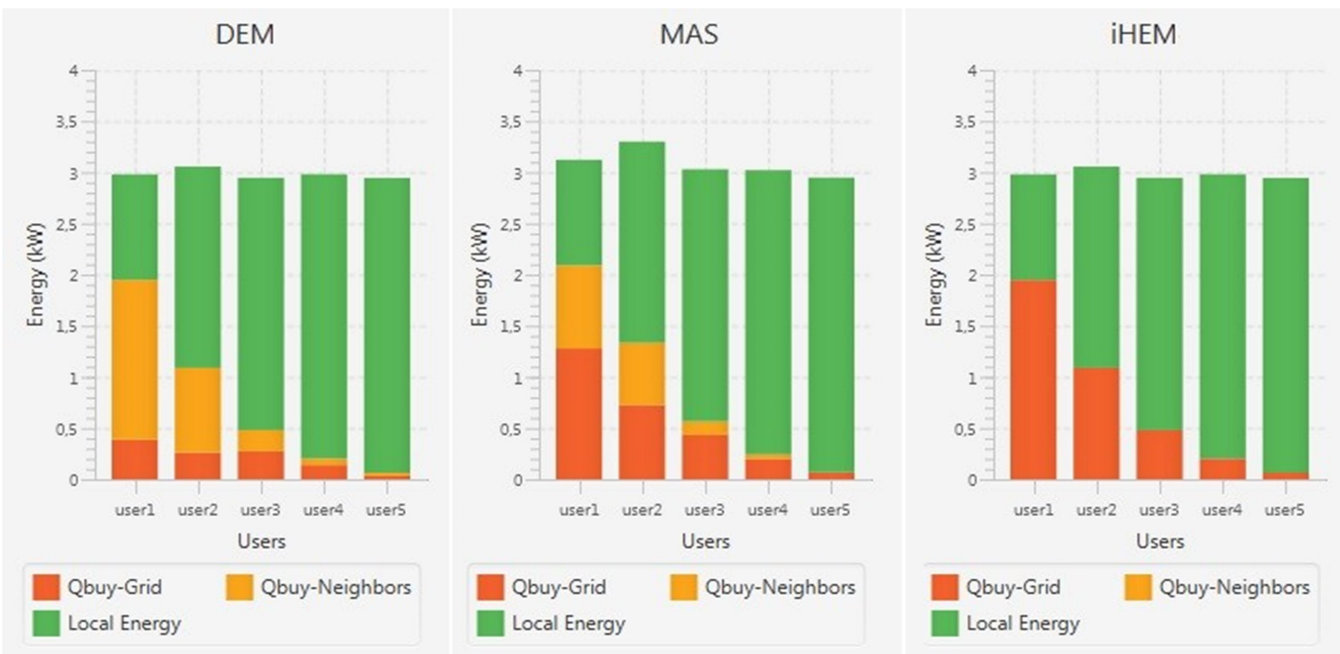

Fig. 6. Energy source.
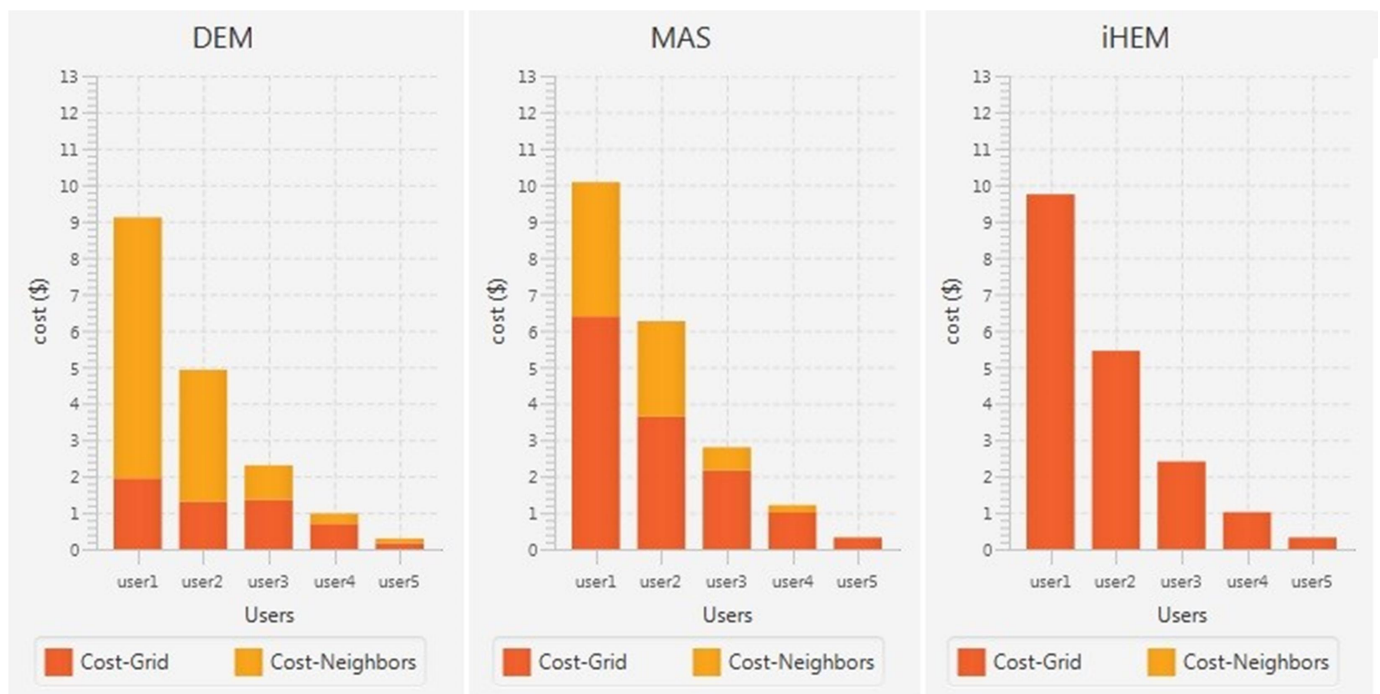

Fig. 7. Expense.

In the following figures (except for «Real expense» results), the yellow color presents all operation with neighbors, the green color presents the amount of energy consumed locally (by local energy source), and the red color presents all operation with Grid (utility companies).

Simulation results show that MAS requests, on average, more quantity of energy than real-time (when an energy request appears) solution (DEM and iHEM) (Fig. 6), because it satisfies each user's demand based on estimation of energy consumption which can be greater, equal or lower to user's real consumption. DEM and iHEM request the same quantity of energy because they satisfy in real-time each user's demand. DEM uses more efficient renewable energy than MAS and iHEM. We observe also that DEM uses less energy from Grid (utility) than MAS and iHEM. Consequently, DEM's users are more able to satisfy their demands on 
energy from local renewable energy. Note that for a user, it is always more profitable to use its own local energy then the energy from the neighbors and at last the energy from the Grid (utility companies). Then, users of DEM expense less than MAS and iHEM (Fig. 7). Depending on the defined trust factor, users of DEM avoid trading with unreliable sellers. Whereas, the users of MAS trade with unreliable sellers, which they lead to satisfy their needs from the grid (at a higher price). DEM sells, on average, less energy to Grid (utility) and more energy to neighbors than iHEM and MAS
(Fig. 8). MAS sells more energy to the Grid because users of MAS may request more quantity of energy than they need. Thus, they expense more than they gain. Fig. 9 shows the gain of each user. iHEM and DEM use a real-time decision (when an energy request appears) that may give a stable (or certain) consumption and production pattern, which causes a transition to a highly efficient economy. Furthermore, energy exchange between neighbors must also be considered (like MAS and DEM).
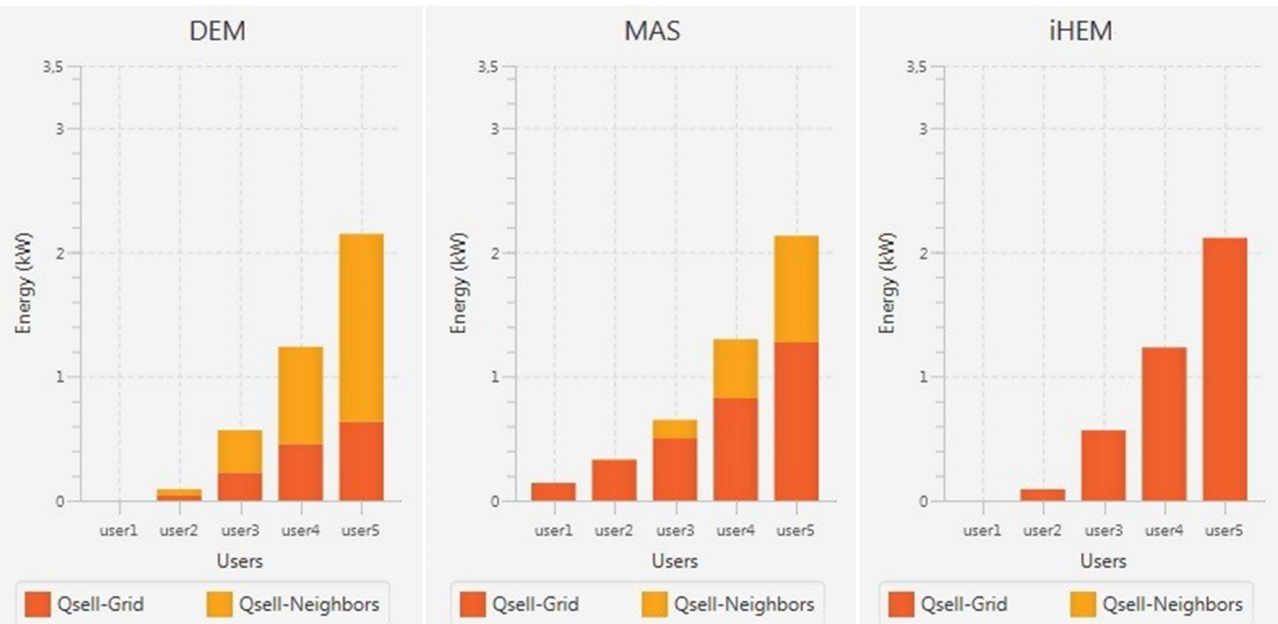

Fig. 8. Quantity sold.

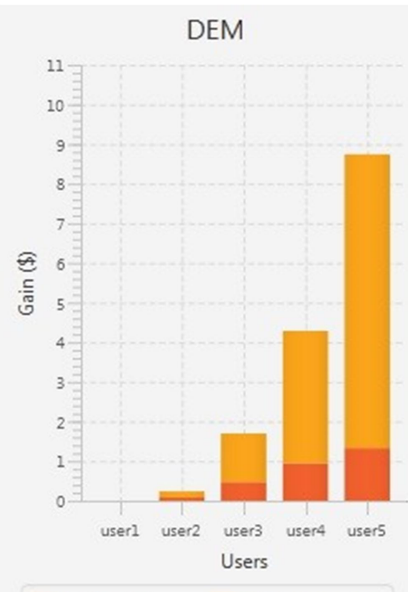

Gain-Grid Gain-Neighbors

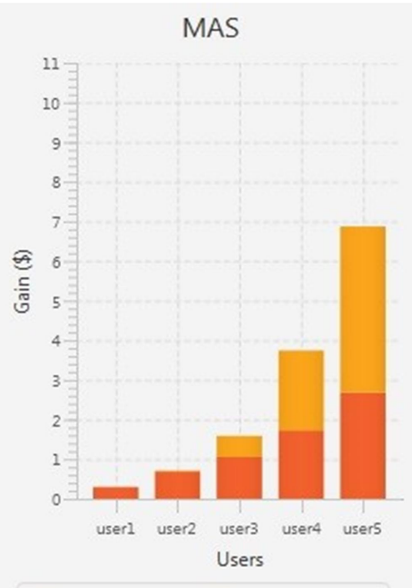

Gain-Grid Gain-Neighbors

Fig. 9. Gain.

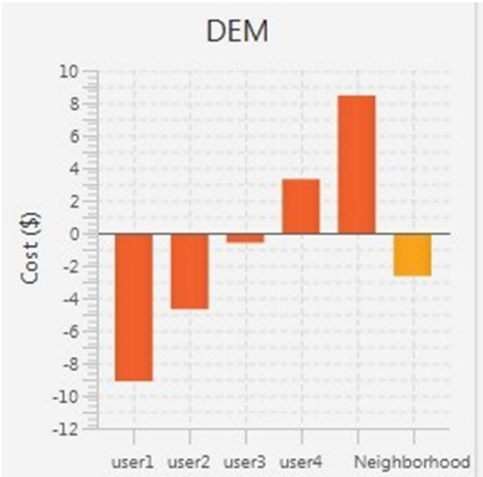

Users

User

Neighborhood: -2.6479237

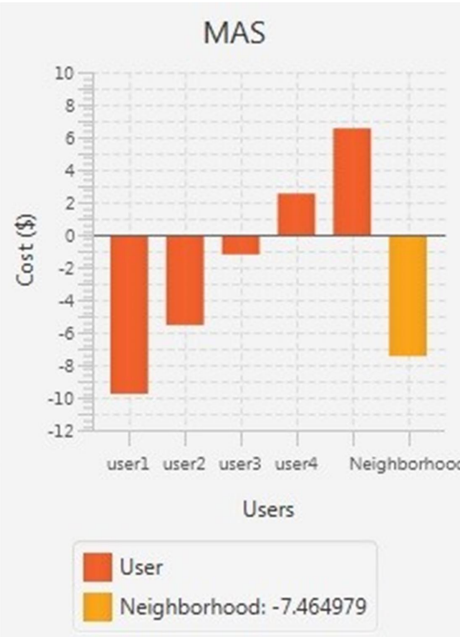

Fig. 10. Real expense.
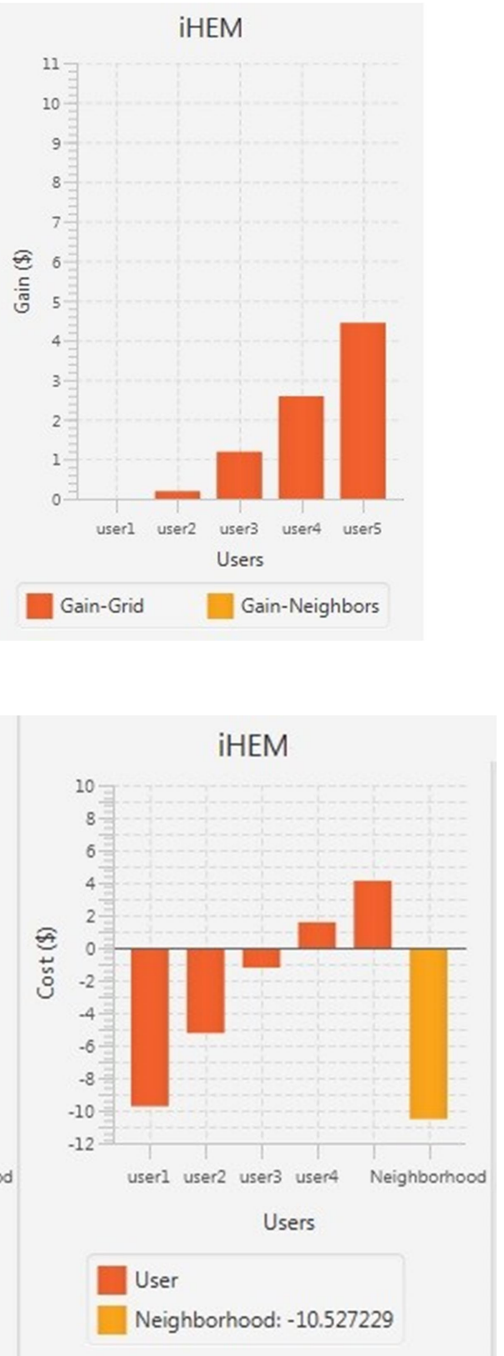
Fig. 10 presents, on average, Real Expense $\left(R E_{n}=\right.$ Gain Expense) for each user $n$ and for the entire neighborhood $\left(R E_{\text {neighborhood }}=\sum R E_{n}\right)$. The sign of $R E_{n}$ determines if it is a benefice $(\geq 0)$ or a deficit $(<0)$.

Fig. 10 shows that the benefice in DEM is greater than in MAS and iHEM and the deficit in DEM is less than in MAS and iHEM (Expense neighborhood $=\$ 2.64$ in DEM, $=\$ 7.46$ in MAS, $=\$ 10.52$ in iHEM (Fig. 10)).

\section{CONCLUSION}

In this paper, we have proposed an intelligent neighborhood based residential energy management approach that takes advantage of neighborhood renewable energy excess. The introduced approach allows users to specify a trust factor on their neighbors in order to determine the best alternative to satisfy their requirements. The defined trust is a good solution to realize optimal energy purchases. Based on the considered trust factor, user may decrease his energy cost by trading with sellers on whom he trusted since they honor their previous promise. After developing the proposed approach, we have evaluated its performance and observed its impact on the neighborhood before deployment. For this purpose, we have introduced a formal approach based on a generic modeling of the global behavior of the studied smart grid. The generation of a set of indicators allows to compare different energy management approaches. The obtained simulation results are very encouraged compared to other approaches.

\section{REFERENCES}

[1] X. Fang, S. Misra, G. Xue, and D. Yang, "Smart grid - the new and improved power grid: A survey," 2011.

[2] R. Walawalkar, S. Fernands, N. Thakur, and K. R. Chevva, "Evolution and current status of demand response (DR) in electricity markets," Insight from PJM and NYISO, 2010

[3] W.-H. Liu, K. Liu, and D. Pearson, "Consumer-centric smart grid," 2011.

[4] M. Erol-Kantarci and H. T. Mouftah, "Wireless sensor networks for cost-efficient residential energy management in the smart grid," IEEE Trans. Smart Grid, vol. 2, no. 12, pp. 314-325, 2011.

[5] A.-H. Mohsenian-Rad and A. Leon-Garcia, "Optimal residential load control with price prediction in real-time electricity pricing environments," IEEE Trans. Smart Grid, vol. 1, no. 12, pp. 120-133, 2010.

[6] M. A. A. Pedrasa, T. D. Spooner, and I. F. MacGill, "Coordinated scheduling of residential distributed energy resources to optimize smart home energy services," IEEE Trans. Smart Grid, vol. 1, no. 12, pp. 134-143, 2010.

[7] E.-K. Melike and T. M. Hussein, "Wireless sensor networks for domestic energy management in smart grids," in Proc. 25th Biennial Symposium on Communications, 2010.

[8] J. M. Lujano-Rojas, C. Monteiro, R. Dufo-Lopez, and A. J. L. BernalAgustin, "Optimum residential load management strategy for real time pricing (RTP) demand response programs," Energy Policy, 2012.

[9] A.-H. Mohsenian-Rad, V. W. Wong, J. Jatskevich, and R. Schober, "Optimal and autonomous incentive-based energy consumption scheduling algorithm for smart grid," presented at the IEEE PES Conf. Innovative Smart Grid Technol., Gaithersburg, MD, 2010.

[10] M. Erol-Kantarci and H. T. Mouftah, "TOU-aware energy management and wireless sensor networks for reducing peak load in smart grids," presented at the Green Wireless Commun. Networks Workshop (GreeNet), IEEE VTC Fall, 2010.

[11] A. Molderink, V. Bakker, M. B. Johann, L. Hurink, and G. J. M. Smit, "Management and control of domestic smart grid technology," IEEE Trans. Smart Grid, vol. 1, no. 12, pp. 109-119, 2010.
[12] W. Zhong, Z. Huang, T. Zhu, Y. Gu, Q. Zhang, P. Yi, D. Jiang, and S. Xiao, "IDES: Incentive-driven distributed energy sharing in sustainable microgrids," In IGC, 2014.

[13] H. S. V. S. K. Nunna and S. Doolla, "Multiagent-based distributedenergy-resource management for intelligent microgrids," IEEE Trans. Ind. Electron., vol. 60, no. 4, pp. 1678-1687, 2013.

[14] Mhadhbi Zeineb, Zairi Sajeh, and Zouari Belhassen, "Generic colored petri nets modeling approach for performance analysis of smart grid system," in Proc. 7th International Renewable Energy Congress (IREC), 2016.

[15] K. Jensen and L. M. Kristensen, "Coloured Petri Nets: modelling and validation of concurrent systems," Springer, 2009.

[16] A. V. Ratzer, L. Wells, H. M. Lassen, et al. "CPN tools for editing, simulating, and analysing coloured petri nets," in Proc. the 24th International Conference on Applications and Theory of Petri Nets (ICATPN 2003), pp. 450-462, 2003.

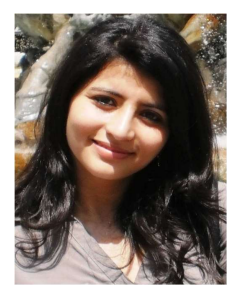

Zeineb Mhadhbi received the master degree in computer science from the University of Tunis El Manar, Faculty of Sciences of Tunis, in 2012. She is currently working toward the Ph.D. degree in the Faculty of Sciences of Tunis. Her research interests are smart microgrids, multiagent system applications, and demand-side management.

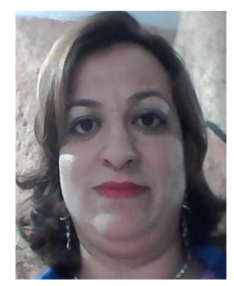

Sajeh Zairi is currently associate professor at the ESEN (Univ. Manouba-Tunisia) and researcher at the Mediatron laboratory (University of Carthage, Tunisia). She was graduated with a Computer Science Engineer degree at FST (Univ. Tunis El Manar- Tunisia), followed by a Joint $\mathrm{PhD}$ in Computer Science and Automation at FST and INSA-Lyon (France). Her main research interest relates to the formal specification and verification of discrete event systems, the optimization of distributed systems, the configuration of sensor network, and the power management in sensor networks and smart grid.

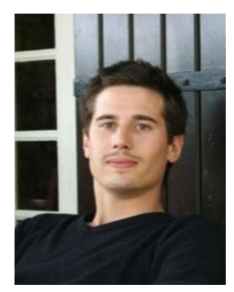

Cedric Gueguen is associate professor at University of Rennes 1 and member of ATNET team at IRISA Labs. Its main research interests are related to wireless network and particularly to scheduling algorithms and green networking. In 2010, he was researcher (ATER) in the department "Protocols Architecture \& Software for Networks" (PASNET) at the University Paris-Est Marne-la-Vallée (LIGM). In November 2010, he obtained his $\mathrm{PhD}$ from the University Pierre et Marie Curie while working in the Networks and Performance nalysis group at the aboratory LIP6. Its PhD thesis focused on the efficient support of multimedia services in OFDM wireless networks, both in the uplink and in the downlink. New access schemes were studied which further increases the benefits of opportunistic scheduling mainly by extending this cross layer technique to higher layers. Before, he hold a M.Sc. in Networking from University Pierre et Marie Curie (UPMC), with a major in performance evaluations.

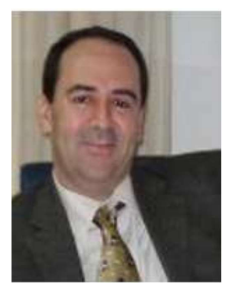

Belhassen Zouari is a professor in computer science at the Higher School of Communications Sup'Commember of Mediatron Lab-University of Carthage, Tunisia. His activities include research works in the fields of verification of automated systems and security issues. He worked on formal verification methods based on coloured Petri nets and applied the related techniques in various fields as Flexible Manufacturing Systems, Wireless Sensor Networks and Business Process Management. He obtained a Doctorate (French PhD) from the University of Paris6 (1993) in Computer Science, and a "Habilitation Universitaire" from the University of Tunis (2005). 